\title{
Energy Harvesting in Wireless Sensor Networks
}

\author{
Anamika Goswami ${ }^{1}$, Mahendra Kumar ${ }^{2}$, Ankita Bajpai ${ }^{3}$ \\ M. tech Scholar, Department of Electronics and Communication Engineering, B.I.E.T., Jhansi, U.P., India ${ }^{1,3}$ \\ Associate Professor, Department of Electronics and Communication Engineering, B.I.E.T., Jhansi, U.P., India ${ }^{2}$
}

\begin{abstract}
Wi-fi or WSN sensor networks are potentially one of the mainly significant technologies of this century. Current development in wi-fi communications and electronics has enable the development of low-value, low-energy, multifunctional minute devices for use in remote sensing applications. The mixture of these elements have stepped forward the viability of using a sensor community which includes a huge variety of clever sensors, allowing the gathering, processing evaluation and dissemination of precious facts amassed in a selection of environments. Sensor community protocols and algorithms have to own self-organizing abilities. Every other specific function of sensor networks is the cooperative attempt of sensor nodes. Sensor nodes are appropriate with an onboard processor. As opposed to sending the uncooked records to the nodes accountable for the fusion, they use their processing talents to domestically perform easy computations and transmit handiest the specified and in part processed records.
\end{abstract}

Keywords: WSN, LEACH, Dead Nodes.

\section{INTRODUCTION}

Recent advancement in electronics has enabled the development of low-cost, low-energy multifunctional miniature devices to be used in remote sensing applications. Such sensors can be extensively deployed for business, a civil and military package which includes surveillance, vehicle tracking, weather and habitat tracking intelligence, medical and acoustic statistics amassing. A WSN consists of large variety of sensor nodes which include sensing, records processing and conversation competencies [1]. Commonly sensor nodes are scattered within the sensing area. They coordinate amongst themselves to get records about the physical surroundings. The records are routed to the bottom station both directly or through other sensor nodes. The Base Station is both a set and cellular node that is capable to connect the sensor community to the internet in which user can get admission to and technique facts. The important thing in sensor networks is to maximize the life of sensor nodes due to the truth that it is not feasible to replace the batteries of thousands of sensor nodes. Consequently, computational operations of nodes and communique protocols ought to be made as energy efficient as viable.

Wi-Fi sensor networks are doubtlessly one of the maximum important eras of this century. Brand new development in wireless communications and electronics has enabled the development of low-cost, low-electricity, multifunctional miniature devices to be used in far off sensing packages. The mixture of these factors have advanced the viability of the use of a sensor community which include a large variety of smart sensors, allowing the collection, processing evaluation and dissemination of valuable records accrued in a selection of environments. A sensor network consists of a large range of sensor nodes which include sensing, information processing and communication talents [2-4].

Sensor network protocols and algorithms have to own self-organizing abilities. Every other precise feature of sensor networks is the cooperative attempt of sensor nodes. Sensor nodes are suitable with an onboard processor. In preference to sending the uncooked information to the nodes responsible for the fusion, they use their processing skills to regionally carry out easy computations and transmit most effective the desired and partially processed information.

This paper is organized as follows: section 1 consists of brief introduction of Wireless sensor networks and protocols. Section 2 consists of working of LEACH protocol, Section 3 presents the improved or proposed technique and section 4 consists of graphs and simulated results on MATLAB 2015. Hence, paper is concluded in the conclusion after results.

\section{LEACH PROTOCOL}

Routing protocols supplying a most suitable facts transmission route from sensor nodes to sink to store electricity of nodes in the network.Information aggregation plays a critical function in energy conservation of sensor network. records aggregation techniques are used now not only for locating an highest quality course from supply to vacation spot but also to dispose of the redundancy of statistics, considering that transmitting massive quantity of raw information is an strength extensive operation, and as a consequence minimizing the wide variety of data transmission. Also more than one sensors might also feel the same phenomenon, despite the fact that from distinct view and if this records may be reconciled right into an extra meaningful shape as it passes through the community, it will become 
greater useful to a software. Moreover while records aggregation is performing facts are compress as it's far exceeded thru the network, thus occupying less bandwidth. This also reduces the quantity of transmission electricity expended by means of nodes as a result layout of energy-green routing protocol can be taken into consideration as a very hard hassle in Wi-Fi sensor community.

LEACH considers all sensors inside the community have the identical quantity of initial strength i.e. they are homogeneous with appreciate to power which isn't sensible technique. So the nodes which right now speak with the BS (i.e. the $\mathrm{CHs}$ ) will die in advance than the everyday cluster member nodes, lowering the general lifespan of the network [5-7]. To make the community greater power inexperienced we classify the sensors into 3 types: ordinary node, intermediate node and superior node relying on their preliminary power diploma. The superior node has the satisfactory power diploma at the equal time because the regular node possesses the bottom degree of energy. The ordinary nodes may be the cluster members. They ship their statistics to their $\mathrm{CH}$ with the intention to be elected from the institution of intermediate nodes. The machine used to select out $\mathrm{CH}$ is identical as that of LEACH [8]. The intermediate nodes can even experience statistics from the environment. they will combination the sensed and received statistics and each ship it to one of the superior nodes positioned nearer to BS or send without delay to the BS if no such superior node is discovered. The development node communicates immediately with the BS. It moreover senses records and aggregates all the facts it received from specific intermediate nodes with its very own facts. In the end it transmits the information right now to the BS.

\section{PROPOSED TECHNIQUE \& RESULTS}

A variety of simulation works / experiments are occurring within the studies subject of WSN to make routing protocols increasingly more electricity green. Here, we propose a modified model of LEACH known as advanced LEACH that may boom power performance than authentic LEACH. The basic concept worried in increasing power efficiency is to preserve radio communique distance as minimal as possible. The famous technique used to reduce communication distance is the formation of clusters between nodes as opposed to direct conversation but as the space among the $\mathrm{CH}$ and BS cross past a sure level single hop conversation concept of LEACH routing protocol is not appropriate.

Our proposed algorithm gives higher connectivity and successful facts rate as evaluate to LEACH. The motive in the back of this enhancement is multi-hop communique adopted through cluster-heads. As member nodes save electricity via sending records to cluster-head in LEACH as opposed to Base station, in addition in stepped forward LEACH cluster-head at longer distance from Base station transmit statistics to superior nodes toward the bottom station instead of direct transmission to Base station its miles greater powerful electricity efficient routing protocol whilst community diameter is larger. Energy performance of progressed LEACH can be higher complex with the example of linear network having two cluster heads A and B which are communicating to Base station. A is at a distance ' $\mathrm{m}$ ' from B and $\mathrm{B}$ is at a distance $\mathrm{m}$ from the bottom station.

$\mathrm{E}_{\text {dirAB }}=\mathrm{E}_{\text {eleTX }} X \mathrm{~L}_{\mathrm{A}}+\mathrm{E}_{\text {amp }} \mathrm{XL}_{\mathrm{A}} \mathrm{X} 2 \mathrm{~m}^{2}+\mathrm{E}_{\text {eleTX }} \mathrm{X} \mathrm{L}_{\mathrm{B}}+\mathrm{E}_{\text {amp }} \mathrm{XL}_{\mathrm{B}} \mathrm{X} \mathrm{m}^{2}$

Working is summed in two phases:

\subsection{Setup Phase:}

1. There are 3 forms of nodes: normal, intermediate and advanced having exclusive energy degrees.

2. To start with Cluster-heads are decided on as in LEACH from the set of intermediate nodes.

3. After cluster formation every $\mathrm{CH}$ will broadcast a quick message containing its identification to find its neighbors.

4. Each non-cluster-head node from the set of regular nodes determines to which cluster it belongs through selecting the cluster-head that calls for the minimal conversation energy, based on the acquired signal energy of the advertisement from every cluster-head.

5. The selected $\mathrm{CH}$ will create a TDMA agenda defining the time slot for every member in its cluster to ahead information to it.

\subsection{Steady-state Phase:}

Like LEACH all of the cluster contributors (normal nodes) will send facts to their corresponding cluster-heads (intermediate node).In contrast to LEACH after aggregation cluster-heads will send the aggregated statistics to a sophisticated node that is closer to the BS than the $\mathrm{CH}$. To discover this type of node $\mathrm{CH}$ will evaluate the gap between advanced node and BS with that among itself and the BS. Whichever is smaller will be used to transmit facts to the BS. If no such advanced node is determined then it'll send the data directly to the base station.

The advance nodes will once more mixture the sensed records and the records acquired from the CHs. After that it's going to ahead the result to the bottom station. 


\subsection{Simulation Results:}

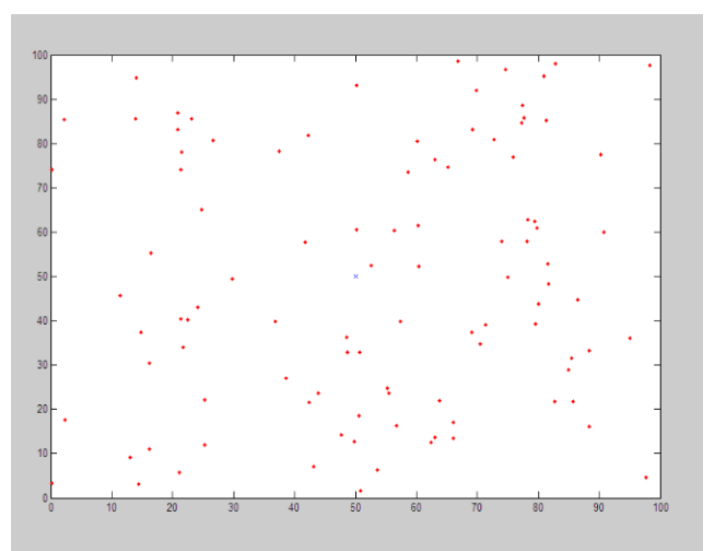

Fig.1All sensor Nodes Dead

Fig. 1 shows formation of dead nodes in simulation represented with red color.

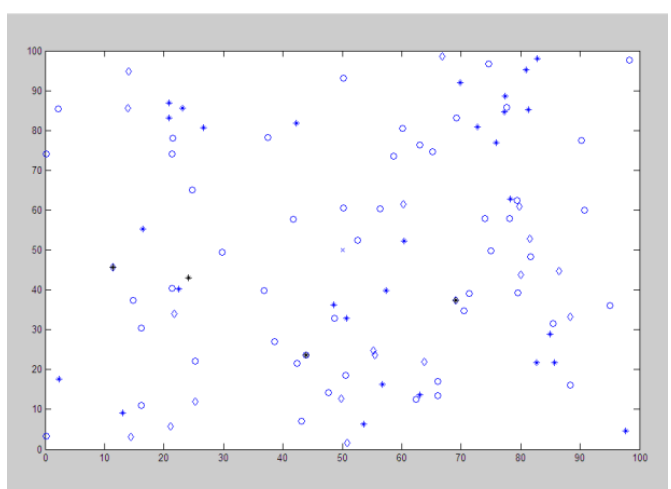

Fig.2Sensor nodes employment across WSN
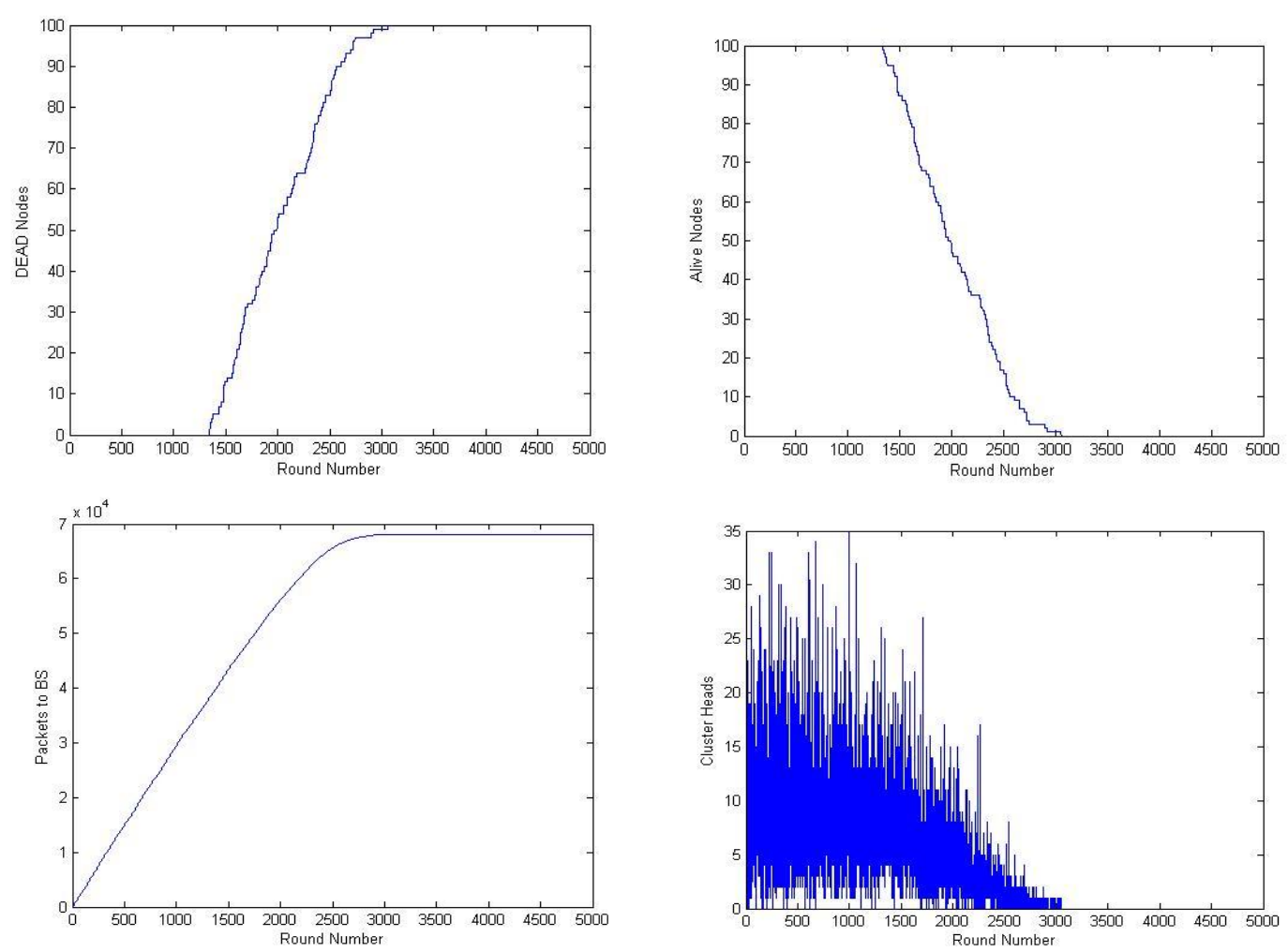

Fig 3.LEACH PROTOCOL 

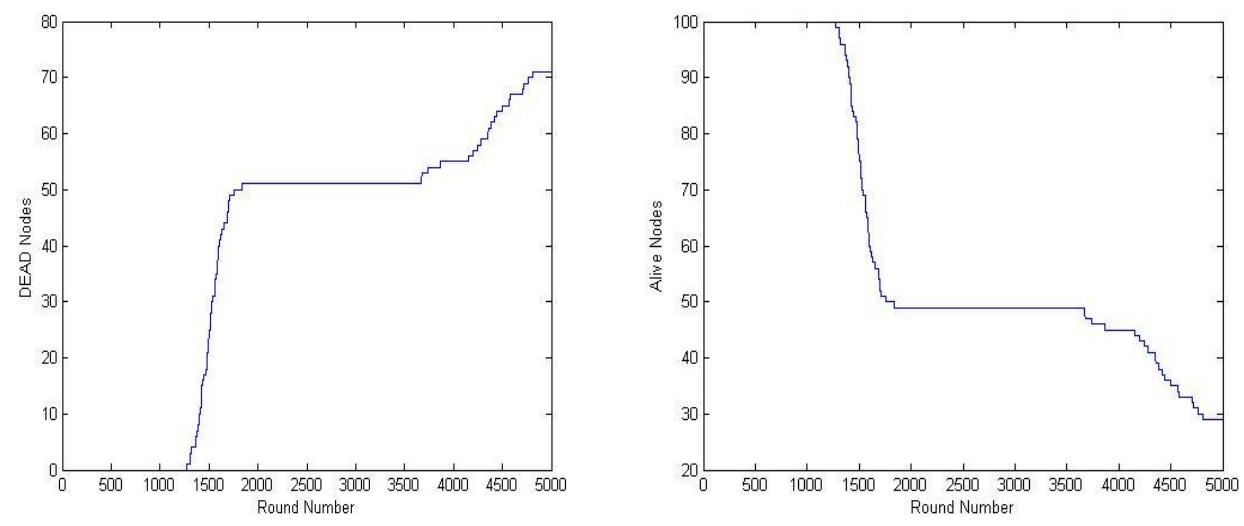

Fig.4(a)Improved LEACH Protocol
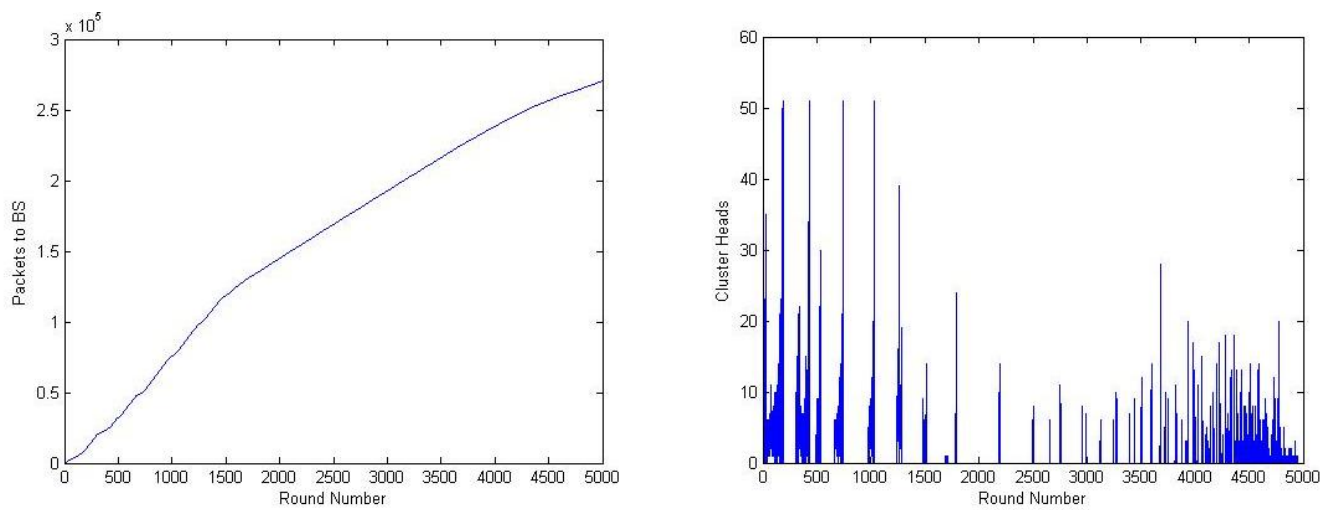

Fig.4(b)Improved LEACH Protocol

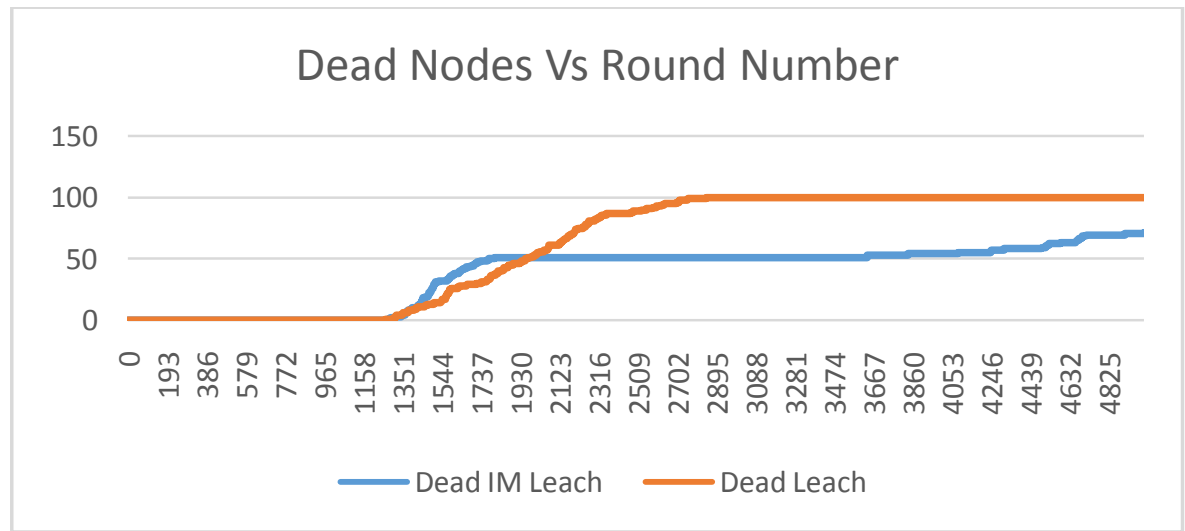

Fig 5. Comparative analysis of LEACH and IMPROVED LEACH

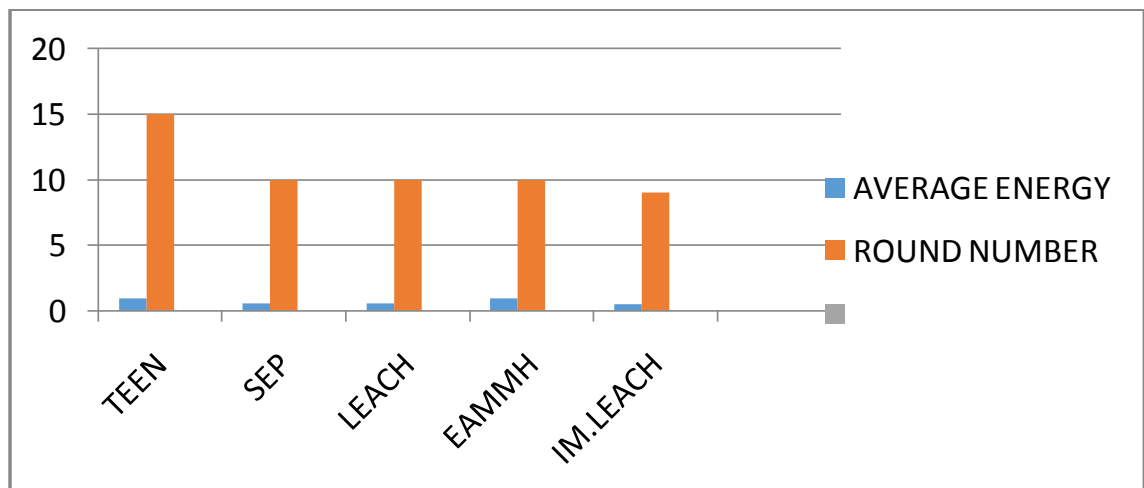

Fig.6 Comparison for Average Energy in different protocols 
Fig.2 gives nodes arrangement on WSN and Fig.3 gives alive nodes, dead nodes cluster head and throughput information for LEACH and Fig. 4 gives information for Improved Leach or proposed. It is clearly seen that the energy efficiency is increased as in improved the no. of nodes are alive for more time and throughput is increased.Fig. 5 shows comparative analysis.

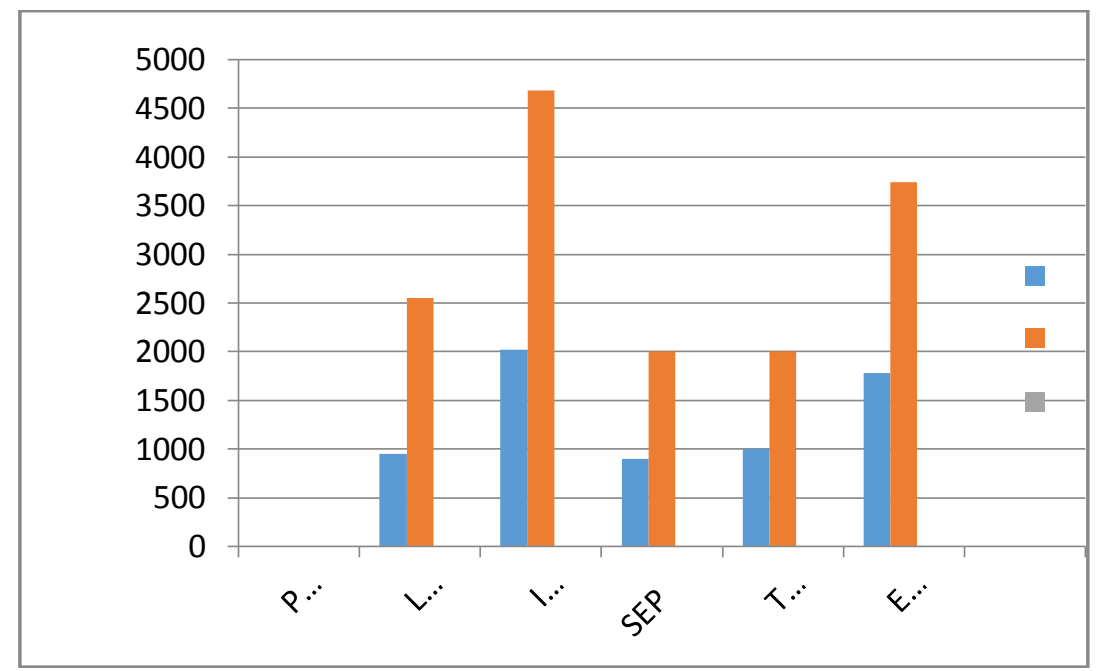

Fig.7 Comparison Dead and Alive Nodes in different protocols

\section{CONCLUSION}

Use of the wireless channel is growing at an amazing speed. Advances in energy-efficient design have created new portable devices that enable exciting applications for the wireless channel. While the wireless channel makes deployment task easier, it adds constraints that are not found in a wired environment. Exclusively, the wireless channel is bandwidth-limited, and the portable devices that use the wireless channel are typically battery-operated and hence energy-constrained. In addition, the wireless channel is error-prone and time-varying. Therefore, it is important to design protocol and algorithms for wireless networks to be bandwidth and energy-efficient as well as robust to channel errors. The work described in this dissertation shows an energy-efficient routing technique which is mainly suitable for application like environment monitoring where sensor nodes located in nearby region collect similar type of data.

\section{REFERENCES}

[1] "Energy Efficient Algorithms for Wireless Sensor Network” Manikandan.K,Vol. 4,Issue 1, IJARCCE, 2015.

[2] “Towards Energy Efficient Big Data Gathering in Densely Distributed Sensor Networks”'DaisukeTakaishi,Vol 2, IEEE, 2014.

[3] "Energy Efficient Wireless Sensor NetworkForPolyhouse Monitoring” SuprotimSinhaMajumdar, EJAET, 2015.

[4] “An Energy-Efficient Routing Algorithm for Wireless Sensor Networks", Guang Yang, IEEE, 2008.

[5] "Clustering in Wireless Sensor Networks: Performance Comparison of EAMMH and LEACH Protocols using MATLAB “ , NishanthThimmegowda , 2015

[6] "Comparison of Energy Efficient Clustering Protocols in Heterogeneous Wireless Sensor Networks ", RaziehSheikhpour, IJAST, 2011

[7] "Clustering Algorithms for Heterogeneous Wireless Sensor Network: A Survey”, VivekKatiyar, Vol1 ,No 2, IJAER, 2010.

[8] “Analysis of LEACH Energy Parameters” Frank Comeaua, NaumanAslam, Procedia Computer Science 5 p.p 933-938, 2011.

[9] “A Review on Energy Harvesting in Wireless Sensor Networks” AnamikaGoswami ,Mahendra Kumar IJIRSET Vol. 6, Issue 4, April ,20 17. 